\title{
The cAMP signaling pathway regulates Epe1 protein levels and heterochromatin assembly
}

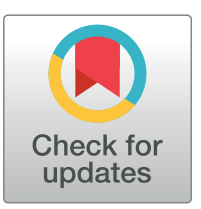

\section{G OPEnACCESS}

Citation: Bao K, Shan C-M, Chen X, Raiymbek G, Monroe JG, Fang Y, et al. (2022) The cAMP signaling pathway regulates Epe1 protein levels and heterochromatin assembly. PLoS Genet 18(2): e1010049. https://doi.org/10.1371/journal. pgen. 1010049

Editor: Michael Freitag, Oregon State University, UNITED STATES

Received: August 30, 2021

Accepted: January 20, 2022

Published: February 16, 2022

Peer Review History: PLOS recognizes the benefits of transparency in the peer review process; therefore, we enable the publication of all of the content of peer review and author responses alongside final, published articles. The editorial history of this article is available here: https://doi.org/10.1371/journal.pgen.1010049

Copyright: ๑ $2022 \mathrm{Bao}$ et al. This is an open access article distributed under the terms of the Creative Commons Attribution License, which permits unrestricted use, distribution, and reproduction in any medium, provided the original author and source are credited.

Data Availability Statement: ChIP-sequencing data that support the findings of this study have been deposited in NCBI GEO with the accession number: GSE181263.

\author{
Kehan Bao $\oplus^{1}$, Chun-Min Shan $\oplus^{10}$, Xiao Chen $\oplus^{2,3}$, Gulzhan Raiymbek $\odot^{4}$, Jeremy \\ G. Monroe $\oplus^{5}$, Yimeng Fang $\oplus^{1}$, Takenori Toda ${ }^{1}$, Kristin S. Koutmou $\oplus^{5}$, \\ Kaushik Ragunathan $\oplus^{4}$, Chao Lu $\circledast^{2,3}$, Luke E. Berchowitz $\oplus^{2}$, Songtao Jia $\oplus^{1 *}$
}

1 Department of Biological Sciences, Columbia University, New York, New York, United States of America, 2 Department of Genetics and Development, Columbia University Irving Medical Center, New York, New York, United States of America, 3 Herbert Irving Comprehensive Cancer Center, Columbia University Irving Medical Center, New York, New York, United States of America, 4 Department of Biological Chemistry, University of Michigan, Ann Arbor, Michigan, United States of America, 5 Department of Chemistry, University of Michigan, Ann Arbor, Michigan, United States of America

a Current address: State Key Laboratory of Plant Genomics, Institute of Microbiology, Chinese Academy of Sciences, Beijing, China

* songtao.jia@ columbia.edu

\section{Abstract}

The epigenetic landscape of a cell frequently changes in response to fluctuations in nutrient levels, but the mechanistic link is not well understood. In fission yeast, the JmjC domain protein Epe1 is critical for maintaining the heterochromatin landscape. While loss of Epe1 results in heterochromatin expansion, overexpression of Epe1 leads to defective heterochromatin. Through a genetic screen, we found that mutations in genes of the cAMP signaling pathway suppress the heterochromatin defects associated with Epe1 overexpression. We further demonstrated that the activation of Pka1, the downstream effector of CAMP signaling, is required for the efficient translation of epe $1^{+}$mRNA to maintain Epe1 overexpression. Moreover, inactivation of the cAMP-signaling pathway, either through genetic mutations or glucose deprivation, leads to the reduction of endogenous Epe1 and corresponding heterochromatin changes. These results reveal the mechanism by which the cAMP signaling pathway regulates heterochromatin landscape in fission yeast.

\section{Author summary}

Genomic DNA is folded with histones into chromatin and posttranslational modifications on histones separate chromatin into active euchromatin and repressive heterochromatin. These chromatin domains often change in response to environmental cues, such as nutrient levels. How environmental changes affect histone modifications is not well understood. Here, we found that in fission yeast, the cAMP signaling pathway is required for the function of Epe1, an enzyme that removes histone modifications associated with heterochromatin. Moreover, we found that active cAMP signaling ensures the efficient translation of $e p e 1^{+} \mathrm{mRNA}$ and therefore maintains high Epe1 protein levels. Finally, we show that changing glucose levels, which modulate cAMP signaling, also affect heterochromatin 
Funding: This work was supported by NIH grants R35GM126910 to S.J., and R35GM138181 to C.L., The Sachaefer Research Program to LEB, The Hirschl Family Trust to LEB, NIH grant R35GM124633 to L.E.B, NIH grant R35GM128836 to KK, and NIH grant R35GM137832 to KR. The funders had no role in study design, data collection and analysis, decision to publish, or preparation of the manuscript.

Competing interests: The authors have declared that no competing interests exist. in a way consistent with cAMP signaling-mediated Epe1 protein level changes. As histone-modifying enzymes often require cofactors that are metabolic intermediates, previous studies on the impact of nutrient levels on chromatin states have mainly focused on metabolites. Our results suggest that nutrient-sensing signaling pathways also regulate histone-modifying enzymes in response to nutritional conditions.

\section{Introduction}

Genomic DNA is folded with histones and non-histone proteins into chromatin, and posttranslational modifications of histones play major roles in regulating genome function. Based on histone modification profiles and other characteristics, chromatin is classified into two main categories: euchromatin and heterochromatin. Euchromatin is gene-rich, transcriptionally active, less condensed, and enriched with histones that are hyperacetylated. On the other hand, heterochromatin is gene-poor, transcriptionally silent, more compact, and enriched with histones that are hypoacetylated and methylated at $\mathrm{H} 3$ lysine 9 (H3K9me) or $\mathrm{H} 3$ lysine K27 (H3K27me) [1,2]. These chromatin states are relatively stable, but they also change dynamically in response to environmental stimuli [3,4]. However, the signaling events that transduce outside signals to chromatin are not well understood.

In the fission yeast Schizosaccharomyces pombe, large blocks of heterochromatin form at repetitive DNA elements near centromeres, telomeres, and at the silent mating-type region. Additionally, about two dozen small heterochromatin islands are scattered throughout the genome $[1,2]$. While large heterochromatin domains are relatively stable, heterochromatin islands frequently change in response to diverse environmental conditions. For example, upon nitrogen starvation, heterochromatin islands are dissembled at meiotic genes as cells prepare for sexual differentiation [5]. In addition, at low temperatures, heterochromatin islands change dramatically in an iron-dependent manner to fine-tune the transcription response [6]. Interestingly, challenges caused by certain genetic mutations or drugs also allow the formation of ectopic heterochromatin islands to create epigenetically silenced gene alleles that enable cells to survive $[7,8]$. How heterochromatin changes in response to environmental conditions is not clear, but the myriad of proteins that participate in the formation and erasure of heterochromatin provide ample targets for signaling pathways to relay environmental information.

The formation of heterochromatin in fission yeast depends on diverse pathways that recruit the histone $\mathrm{H} 3 \mathrm{~K} 9$ methyltransferase $\mathrm{Clr} 4$ to distinct genomic locations [1,2]. For example, repetitive DNA elements recruit Clr4 through the RITS (RNA induced transcriptional silencing) complex, which uses small interfering RNAs (siRNAs) generated by the RNA interference (RNAi) machinery as guides to home in on the nascent transcripts produced at repeat regions [9-13]. In addition, DNA binding proteins, such as ATF/CREB proteins Atf1/Pcr1 and shelterin, recruit $\mathrm{Clr} 4$ to nucleate heterochromatin near repeats at the silent mating-type region and telomeres, respectively [14-16]. Moreover, the RNA elimination machinery recognizes nascent RNAs containing determinant of selective removal (DSR) sequence to recruit $\mathrm{Clr} 4$ and establish heterochromatin islands [5]. Clr4-mediated H3K9 methylation leads to the recruitment of HP1 proteins to create a silenced chromatin state that represses both transcription and recombination [1,17-19].

Maintaining the proper heterochromatin landscape also depends on diverse activities that remove heterochromatin from inappropriate sites, such as the Mst2 histone H3K14 acetyltransferase complex, the INO80 chromatin remodeling complex, and the JmjC domain protein Epe1 $[5,7,20]$. Epe1 is a resident heterochromatin protein and is recruited to heterochromatin 
through its interaction with Swi6 [21-23]. It contains a JmjC domain, which is the catalytic domain of histone demethylases [24]. Although no demethylase activity has been demonstrated for Epe1 in vitro [24], in vivo evidence demonstrates that Epe1 is the major "eraser" of H3K9me. For example, loss of Epe1 leads to spreading of heterochromatin outside of its normal boundaries, expansion of heterochromatin islands, formation of ectopic heterochromatin, and more stable inheritance of heterochromatin. In contrast, overexpression of Epe1 leads to the destabilization of existing heterochromatin [5,7,21,22,25-29]. Therefore, Epe1 protein levels need to be tightly regulated within a narrow range. Indeed, Epe1 is a target of the Cul4-Ddb1 ubiquitin E3 ligase, which mediates its degradation by the proteasome [30]. Epe1 is required for heterochromatin island changes in response to nitrogen starvation and loss of Epel function has been implicated in the generation of new epigenetically silenced alleles $[5,7,8,29]$. However, how Epe1 links changes in the heterochromatin landscape to environmental conditions is still unknown.

In this study, we performed a genetic screen to identify regulators of Epel function. We found that an active cAMP signaling pathway is critical for the ability of Epel to erase heterochromatin. We further demonstrated that the cAMP signaling pathway maintains Epe1 protein levels by regulating its mRNA translation. These results provide a critical link between nutritional conditions and the heterochromatin landscape of the genome.

\section{Results}

\section{The cAMP signaling pathway regulates Epe1 function}

Given the critical role of Epe1 in modulating the heterochromatin landscape, we performed a genetic screen for regulators of Epel function. We replaced the endogenous promoter of epe $1^{+}$ with an $n m t 41$ promoter, which can be induced to overexpress Epe1 when cells are grown in a medium without thiamine (Edinburgh minimal medium, EMM) [31]. We also used a reporter gene inserted within pericentric repeats (otr:ura ${ }^{+}$) to measure heterochromatin integrity [32]. In wild-type cells, otr:ura $4^{+}$is silenced by the formation of heterochromatin, resulting in cells resistant to 5-fluoroorotic acid (5-FOA). When Epe1 is overexpressed, heterochromatin is compromised, leading to the expression of the otr:ura $4^{+}$reporter and thus poor cell growth on media containing 5-FOA [21,22,28].

We crossed a query strain containing both the otr::ura $4^{+}$reporter and $n m t 41$-epe $1^{+}$with the fission yeast deletion library, which contains $\sim 3500$ nonessential gene deletions (Fig 1A). After selecting haploid progeny containing otr::ura4 ${ }^{+}, n m t 41-e p e 1^{+}$, and the deletion of a single gene, cells were grown on EMM medium containing 5-FOA to measure the silencing of otr:: $\mathrm{ura}^{+}$. We found that deletions of several genes involved in the cAMP (cyclic adenosine 3',5'monophosphate)-signaling pathway, such as git $1 \Delta$, git $3 \Delta$, git $5 \Delta$, gpa $2 \Delta$, and pka $1 \Delta$, rescue the silencing defect caused by Epe1 overexpression (Fig 1B).

The cAMP signaling pathway coordinates cellular responses with outside stimuli, such as hormones and nutrients, and is largely conserved from yeast to mammals [33,34] (Fig 1C). In fission yeast, ligand molecules bind to the transmembrane $\mathrm{G}$ protein-coupled receptor (GPCR) Git3, which in turn activates the G protein trimers Gpa2/Git5/Git11, releasing the Galpha subunit Gpa2 to activate the adenylyl cyclase Cyr1 to synthesize cAMP. Protein kinase A (Pka1) is the major effector of the cAMP signaling pathway in fission yeast. In the absence of cAMP, Pka1 is inactive due to its association with a regulatory subunit, Cgsl. In the presence of cAMP, Cgs1 dissociates from Pkal. Pka1 then translocates to the nucleus and phosphorylates its substrates. The identification of multiple mutants of the cAMP signaling pathway in our screen indicates that this pathway regulates Epel function. 
A

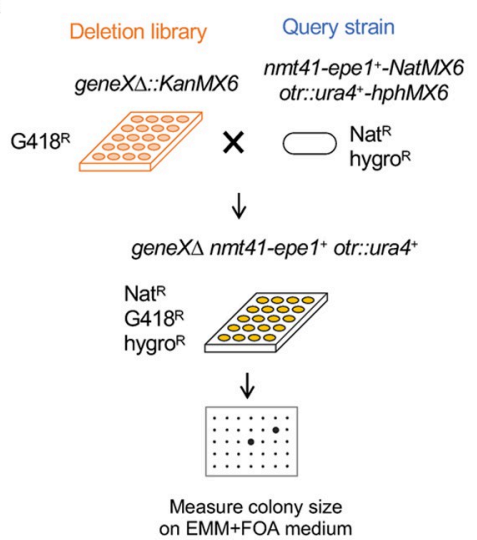

B

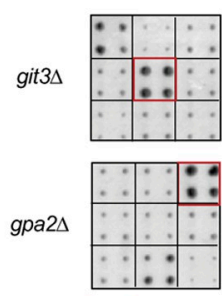

git5s

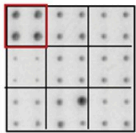

git1 $\Delta$

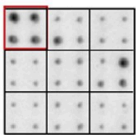

pka1s

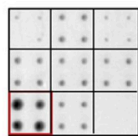

D

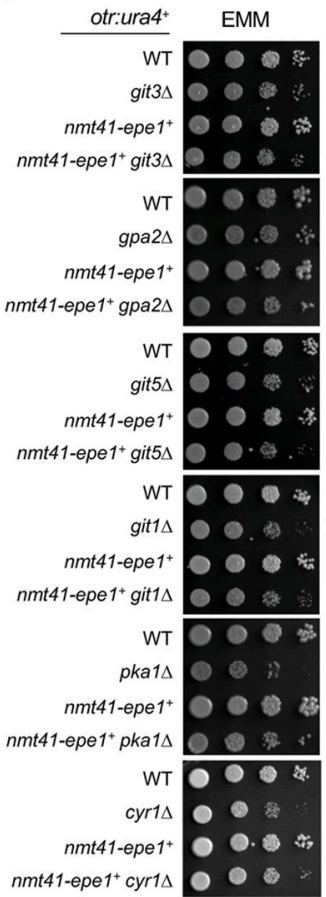

C

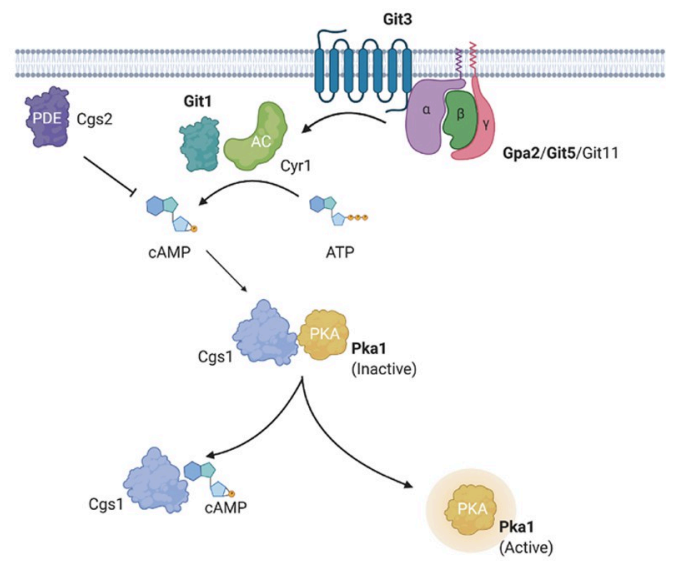

E

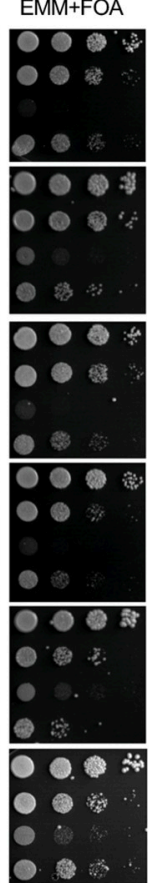

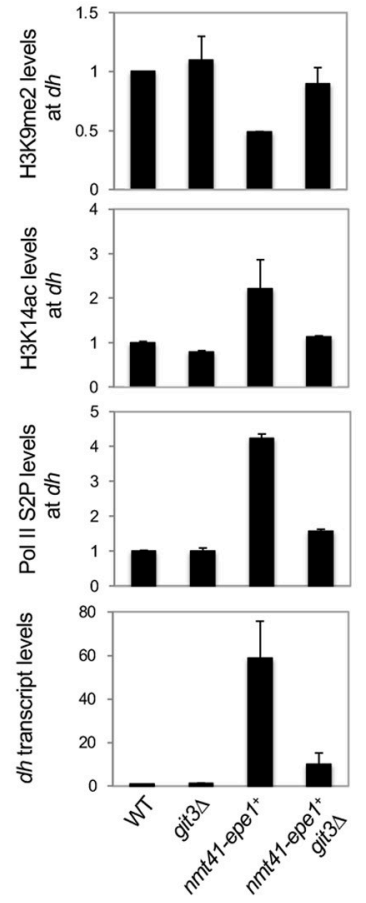

Fig 1. A genetic screen for regulators of Epe1 function. (A) Workflow to introduce otr::ura4 ${ }^{+}$and $n m t 41$-epe $1^{+}$into the deletion library. (B) Representative images of cells grown on EMM+FOA medium. Each square represents one gene deletion in quadruplicates. Red boxes represent cells with genotypes marked on the left. (C) Diagram of the cAMP signaling pathway in $S$. pombe. The factors identified in our screen are in bold. (D) Ten-fold serial dilution analyses of indicated yeast strains grown on indicated media to measure the expression of the otr::ura4 ${ }^{+}$reporter. (E) ChIP analyses of H3K9me2, H3K14ac, and Pol II S2P levels at pericentric repeats $(d h)$, normalized to leu $1^{+}$, and RTqPCR analysis of pericentric transcript $(d h)$, normalized to $a c t 1^{+}$. Error bars represent SD, $\mathrm{n} \geq 3$.

\section{https://doi.org/10.1371/journal.pgen.1010049.g001}

To validate the results of our genetic screen, we reconstructed cells containing otr::ura $4^{+}$ $n m t 41-e p e 1^{+}$and individual gene deletions by genetic crosses. We also constructed cyrlu, which is not present in the deletion library. Consistent with the results of the genetic screen,

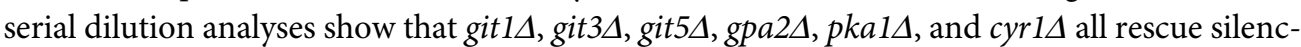
ing defects of otr::ura $4^{+}$caused by Epe1 overexpression, as indicated by better growth on EMM medium containing 5-FOA (Fig 1D). 
We then examined the effects of cAMP signaling on heterochromatin at pericentric repeats, which are divided into $d h$ and $d g$ regions. Pericentric heterochromatin is enriched in repressive histone posttranslational modifications such as $\mathrm{H} 3 \mathrm{~K} 9$ methylation. Epe1-overexpression not only results in the loss of silencing of reporter genes inserted within pericentric heterochromatin but also a reduction of $\mathrm{H} 3 \mathrm{~K} 9$ methylation levels at $d h$ repeats [21,22,28]. ChIP analysis shows that $\mathrm{H} 3 \mathrm{~K} 9 \mathrm{me} 2$ levels at $d h$ repeats are restored close to wild-type levels in git3u nmt41-epe $1^{+}$cells (Fig 1E).

Epe1 overexpression recruits the SAGA histone acetyltransferase to pericentric repeats, leading to increases in H3K14ac and Ser2-phosphorylated Pol II levels at heterochromatin, as well as increases in $d h$ transcripts levels [28]. ChIP analysis reveals that both H3K14ac and Ser2-phosphorylated Pol II are restored to near wild-type levels in git34 nmt41-epe $1^{+}$cells (Fig 1E). Moreover, RT-qPCR analysis shows that the pericentric repeat transcript levels are elevated in nmt41-epe $1^{+}$cells, but are reduced to near wild-type levels in git $3 \Delta$ nmt41-epe $1^{+}$cells (Fig 1E). These results support a model whereby active cAMP signaling mediates the heterochromatin defects caused by Epe1 overexpression.

\section{Intracellular cAMP levels regulate Epe1 function}

We next examined whether intracellular cAMP levels regulate Epe1 function. In fission yeast, the transmembrane G-protein-coupled receptor Git3 and the downstream heterotrimeric G proteins (Gpa2, Git5, and Git11) activate the adenylate cyclase Cyr1 to raise intracellular cAMP levels [33]. Cgs2 is a phosphodiesterase that breaks down cAMP, and $\operatorname{cgs} 2 \Delta$ raises intracellular cAMP levels in the absence of Git3 [35] (Fig 2A). Interestingly, although git3 $n m t 41$-epe $1^{+}$cells form heterochromatin at pericentric repeats, cgs $2 \Delta$ git $3 \Delta$ nmt41-epe $1^{+}$cells do not, as indicated by both serial dilution analysis to measure the expression of otr::ura $4^{+}$and RT-qPCR analysis to measure $d h$ transcript levels (Fig 2B and 2C). These results suggest that the reduction of cellular cAMP levels caused by git3 $\Delta$ is responsible for the rescue of the Epe1 overexpression phenotype.

To further examine the role of cAMP in this process, we directly added cAMP to the growth medium. RT-qPCR analysis shows that $d h$ transcript levels of git $3 \Delta$ nmt41-epe $1^{+}$cells are almost at wild-type levels when grown in EMM medium, but they are higher in the presence of $5 \mathrm{mM}$ exogenous cAMP (Fig 2D), consistent with the notion that cAMP levels regulate Epe1 function.

\section{Activation of Pkal is required for Epel function}

We then examined whether cAMP affects Epel function via the effector kinase Pka1. When cAMP levels are low, Pkal is inactive due to the association of its regulatory subunit Cgs1. In cgs $1 \Delta$ cells, Pka1 is constitutively active regardless of intracellular cAMP levels [36] (Fig 2A). Similar to $c g s 2 \Delta$, cgs $1 \Delta$ also reverts the rescue of heterochromatin in git $3 \Delta$ nmt41-epe $1^{+}$cells, as measured by serial dilution analysis to measure otr::ura4 ${ }^{+}$expression and RT-qPCR analysis to measure $d h$ transcript levels (Fig 2B and 2C). In contrast, $\operatorname{cgs} 2 \Delta, \operatorname{cgs} 1 \Delta$, or the addition of cAMP have mild effects on $d h$ transcript levels in pka14 nmt41-epe $1^{+}$cells (Fig 2E). These results support the idea that cAMP activates Pka1, which in turn stimulates Epel function.

\section{cAMP signaling pathway regulates Epe1 protein levels}

To further examine how the cAMP signaling pathway regulates Epel function, we assessed the effects of cAMP signaling mutations on Epe1 protein levels. Interestingly, we found that Epe1

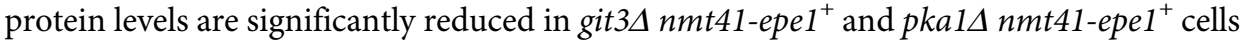
(Fig 2 F). In addition, Epe1 protein levels are largely restored to wild-type levels in git3 $\Delta$ cgs1 $\Delta$ 
A

\begin{tabular}{|c|c|c|}
\hline Genotype & cAMP level & Pka1 Status \\
\hline WT & High & Active \\
\hline git $3 \Delta$ & Low & Inactive \\
\hline git $3 \Delta$ cgs $2 \Delta$ & High & Active \\
\hline git $3 \Delta$ cgs $1 \Delta$ & Low & Active \\
\hline git $3 \Delta+$ cAMP & High & Active \\
\hline
\end{tabular}

$c$

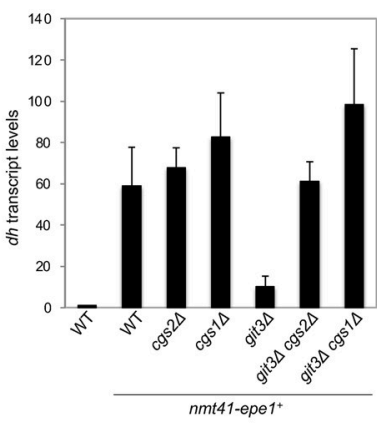

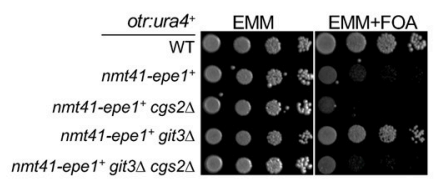

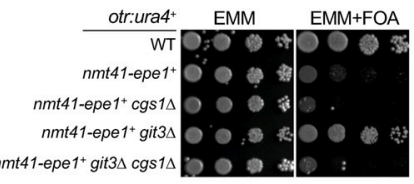

D

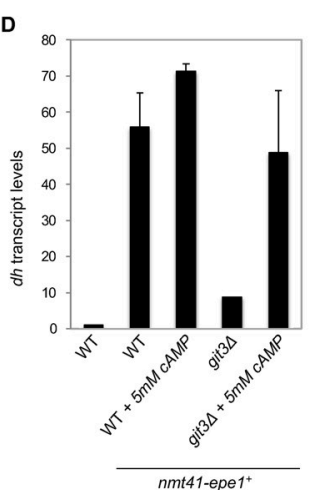

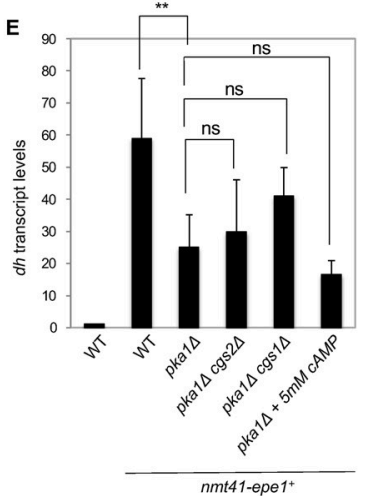

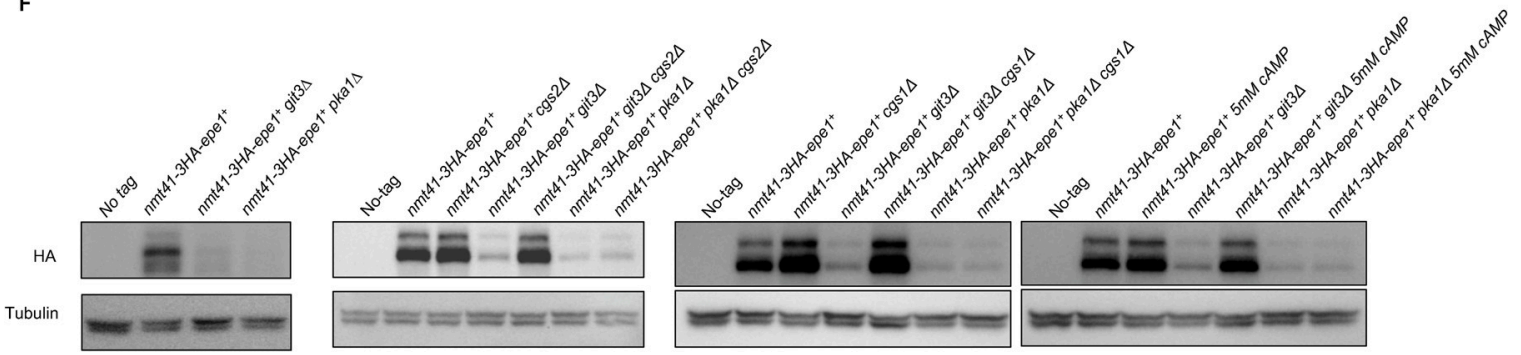

Fig 2. High levels of cAMP and an active Pka1 are required for Epel function. (A) Summary of effects of $g i t 3 \Delta, c g s 2 \Delta, c g s 1 \Delta$, and exogenous cAMP on cellular cAMP levels and the activity of Pka1. (B) Ten-fold serial dilution analyses of indicated yeast strains grown on indicated media to measure the expression of the otr::ura $4^{+}$reporter gene. (C-E) RT-qPCR analyses of pericentric $d h$ transcript, normalized to $a c t 1^{+}$. Error bars represent SD, $\mathrm{n} \geq 2$. One-way ANOVA was performed comparing each strain to $n m t 41$-epe $1^{+} p k a 1 \Delta$. (F) Western blot analyses to measure the levels of 3HA-tagged Epe1 under the control of nmt41 promoter and the levels of tubulin.

https://doi.org/10.1371/journal.pgen.1010049.g002

$n m t 41-e p e 1^{+}$and git3 $\Delta$ cgs $2 \Delta$ nmt41-epe $1^{+}$cells (Fig 2F). Moreover, $5 \mathrm{mM}$ exogenous cAMP also restores Epe1 protein levels in git $3 \Delta$ nmt41-epe $1^{+}$cells (Fig 2F). On the other hand, cgs1 , cgs $2 \Delta$, and $5 \mathrm{mM}$ exogenous cAMP do not restore Epe1 levels in pka1 $\Delta$ cells (Fig 2F). These data suggest that the CAMP signaling pathway governs Epe1 protein homeostasis.

\section{Epel phosphorylation by Pka1 does not contribute to Epel protein level control}

One possible explanation of how cAMP signaling regulates Epel protein levels is that Epe1 is a direct target of Pkal phosphorylation. Indeed, we found that Pkal phosphorylates recombinant Epe1 in vitro, although the activity is much weaker compared to another Pka1 substrate Rst2 (S1A and S1B Fig). We subjected in vitro phosphorylated Epe1 to mass-spec analysis and identified S717 as the only phosphorylation site. We mutated S717 to a phosphomimetic amino acid (S717D), but this mutation did not protect Epe1 levels from decreasing in git3 $\Delta$ cells (S1C Fig). In addition, bioinformatics analysis also predicted Pka1 phosphorylation sites at residues $\mathrm{S} 606$ and T607. We mutated these two residues to phosphomimetic amino acids 
(S606D T607D), but the protein levels of this Epe1 mutant also decrease in git3A cells (S1D Fig). These results suggest that Pka1-mediated phosphorylation of Epe1 might not be responsible for Epe1 protein level changes in the absence of active cAMP signaling.

\section{cAMP signaling pathway regulates Epe1 protein levels through translation control}

The cAMP signaling pathway may also regulate Epe1 protein levels through transcription, translation, or protein degradation. To distinguish these possibilities, we first measured $e p e 1^{+}$ transcript levels by RT-qPCR. However, we did not observe any reduction of $e p e 1^{+}$mRNA levels in git $3 \Delta$ or pka1 $1 \Delta$ cells (Fig $3 \mathrm{~A}$ ), indicating that cAMP signaling regulates Epe1 protein levels through a post-transcriptional mechanism.

Because Epe1 protein levels are regulated by the Cul4-Ddb1 E3 ubiquitin ligase complex [30], we tested whether cAMP signaling regulates Cul4-Ddb1-mediated Epe1 degradation. There are severe growth defects associated with $d d b 1 \Delta$ due to the accumulation of Spd1, one of Ddbl's targets [37]. Therefore, we used $d d b 1 \Delta$ spd $1 \Delta$ cells to avoid complications from slow growth. Epe1 protein levels remain low in git3 $\Delta d d b 1 \Delta$ spd $1 \Delta$ cells, similar to those in git3 $\Delta$ cells, suggesting that the cAMP signaling pathway does not regulate Epe1 degradation through Cul4-Ddb1 (Fig 3B).

To further examine whether cAMP signaling regulates Epe1 degradation, we measured Epe1 degradation kinetics in wild-type and git $3 \Delta$ cells after the addition of cycloheximide

A

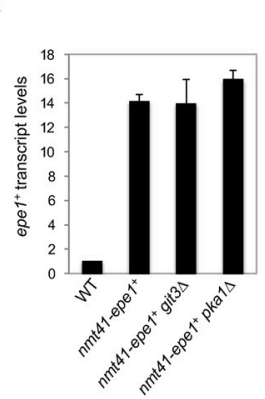

D
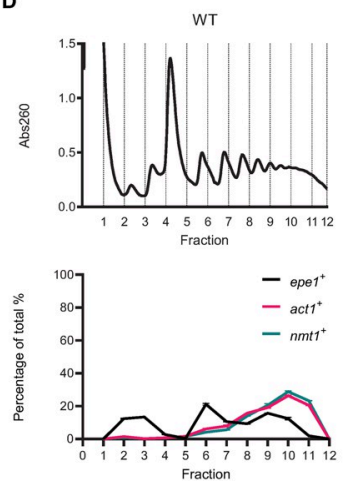

B
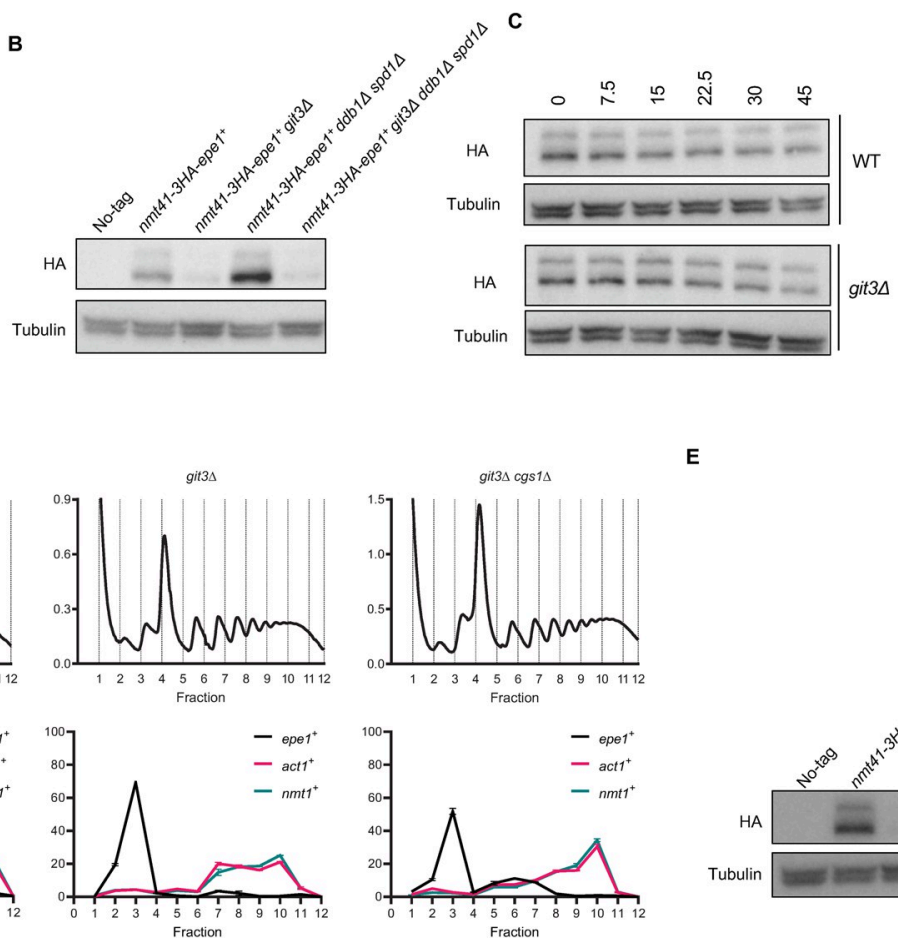

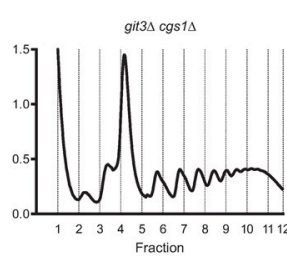

E
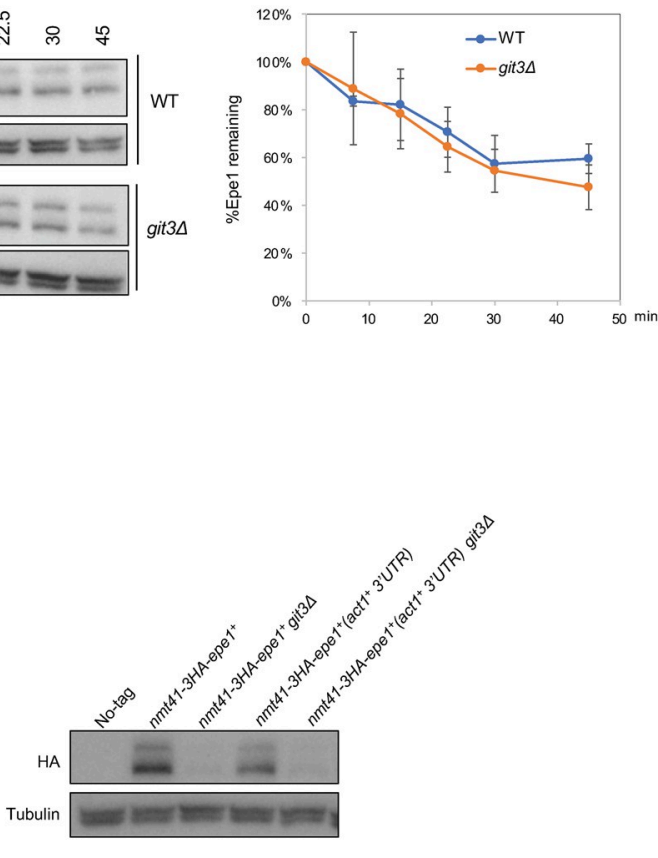

Fig 3. The cAMP signaling pathway regulates the translation of $e p e 1^{+}$mRNA. (A) RT-qPCR analysis of $e p e 1^{+}$transcript, normalized to $a c t 1^{+}$. Error bars represent $\mathrm{SD}, \mathrm{n}=2$. (B, E) Western blot analysis to measure the levels of 3HA-tagged Epe1 under the control of $n m t 41$ promoter and levels of tubulin. (C) Left, western blot analysis to measure the levels of 3HA-tagged Epe1 under the control of $n m t 41$ promoter and tubulin. Samples are taken at indicated time points (minutes) after the addition of cycloheximide. Different exposures were used for wild type and git3 $\Delta$ cells to make the signal at time 0 comparable. Right, quantification of Epel proteins levels. Error bars represent SD, n $=2$. (D) Top, sucrose density gradients of ribosomes in each strain with continuous monitoring of absorbance at $260 \mathrm{~nm}$. Lighter fractions are on the left. Bottom, qRT-PCR analysis of $e p e 1^{+}$, act $1^{+}$, and $n m t 1^{+}$ transcripts from each fraction. Relative amounts of transcripts were calculated using the delta Ct method. The distribution is shown as a percentage of the total. Error bars represent the SD of two technical replicates.

https://doi.org/10.1371/journal.pgen.1010049.g003 
A

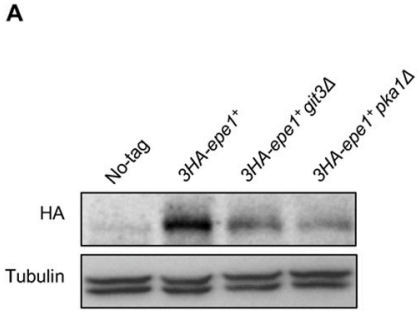

D

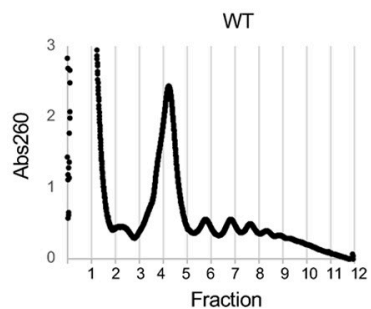

B
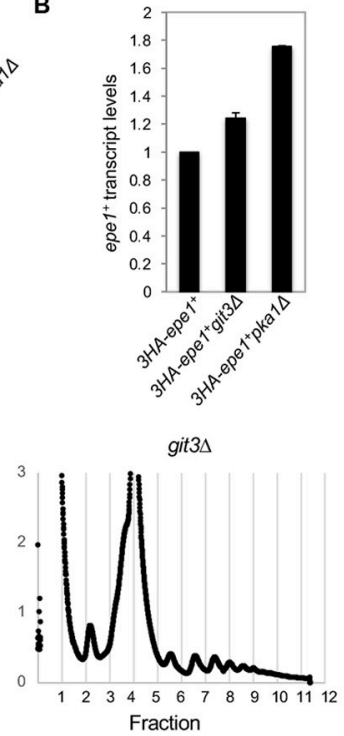

C

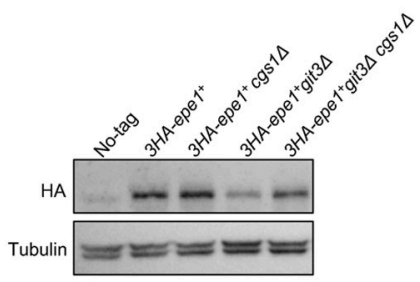

$E$

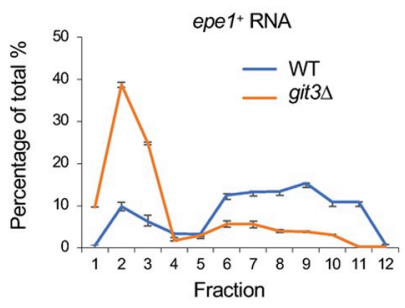

Fig 4. The cAMP signaling pathway regulates the protein levels of endogenous Epe1. (A, C) Western blot analysis to measure the levels of endogenous 3HA-tagged Epe1 and tubulin. (B) RT-qPCR analysis of $e p e 1^{+}$transcript levels, normalized to $a c t 1^{+}$. Error bars represent SD. $\mathrm{n}=2$. (C) Sucrose density gradients of ribosomes in each strain with continuous monitoring of absorbance at $260 \mathrm{~nm}$. Lighter fractions are on the left. (E) qRT-PCR analysis of epe $1^{+}$ transcripts from each fraction. Relative amounts of transcripts were calculated using the delta Ct method. The distribution is shown as a percentage of the total. Error bars represent the SD of two technical replicates.

https://doi.org/10.1371/journal.pgen.1010049.g004

(CHX) to block new protein synthesis. The degradation rates of Epe1 are similar over a 45-minute period after CHX addition, indicating that the cAMP signaling pathway does not control Epe1 levels by regulating its degradation (Fig 3C).

We then examined whether cAMP signaling regulates the translation of Epe1 using polysome profiling. In nmt41-epe $1^{+}$cells grown in EMM medium, epe $1^{+}$mRNA is broadly distributed into different fractions. In contrast, an actively transcribed housekeeping gene $a c t 1^{+}$, which encodes actin, is mainly present in the polysome fractions (5 through 12) (Fig 3D). Remarkably, git $3 \Delta$ nearly abolished $e p e 1^{+}$mRNA in the polysome fractions, and git $3 \Delta \operatorname{cgs} 1 \Delta$ partially restores polysome-associated $e p e 1^{+}$mRNA (Fig 3D). These results suggest that cAMP signaling regulates $e p e 1^{+}$mRNA translation.

We then assessed whether and to what degree the cAMP-mediated effects on Epe1 translation were dependent on the untranslated regions (UTRs). The nmt41-epe $1^{+}$construct replaces the endogenous epe $1^{+}$promoter with a $n m t 41$ promoter, which contains a 4 base pair deletion at the TATA box of the endogenous $n m t 1$ promoter to reduce its expression $[31,38]$. The $5^{\prime}$ UTR of $n m t 41-e p e 1^{+}$is identical to that of $n m t 1^{+}$. The endogenous $n m t 1^{+}$mRNA, which is also induced to express at high levels, shows a distribution in polysome profile similar to that of $a c t 1^{+}$mRNA, and the distribution is not severely affected by either git $3 \Delta$ or git $3 \Delta \operatorname{cgs} 1 \Delta$ (Fig 3D). We also replaced the $3^{\prime}$-UTR of $n m t 41-e p e 1^{+}$with the $3^{\prime}$-UTR of $a c t 1^{+}$and found that Epe1 protein levels are still reduced in git3 $\Delta$ cells (Fig $3 \mathrm{E}$ ). These results strongly argue against a role for the $5^{\prime}$-UTR or $3^{\prime}$-UTR regions in regulating $e p e 1^{+}$mRNA translation.

\section{The cAMP signaling pathway regulates endogenous Epel protein levels and heterochromatin}

We then tested if the cAMP signaling pathway regulates Epe1 expressed from the endogenous locus. We inserted three copies of $\mathrm{HA}$ tag at the $\mathrm{N}$-terminus of the $e p e 1^{+}$at its endogenous 
chromosomal locus, keeping the promoter, 5'UTR, and 3'-UTR intact. We first tested whether the addition of the HA tag affects Epe1 function using an $a d e 6^{+}$reporter inserted outside of mating-type region heterochromatin (SacI::ade6 ${ }^{+}$) (S2A Fig). This reporter is fully expressed in wild-type cells, resulting in white colonies when cells are grown on a low adenine medium (YE) [25] (S2B Fig). In epe14 cells, heterochromatin spreads outside of the boundary to silence Saci::ade6 ${ }^{+}$, resulting in red colonies. The addition of the HA-tag does not significantly compromise Epe1 function, as HA-Epe1 expressing cells form mostly white colonies (S2B Fig). In both git $3 \Delta$ and pka1 $\Delta$ cells, HA-Epe1 protein levels are reduced without a corresponding decrease in epe ${ }^{+}$mRNA levels (Fig 4A and 4B). Moreover, Epel protein levels increase in git $3 \Delta$ cgs $1 \Delta$ cells compared with git $3 \Delta$ cells (Fig $4 \mathrm{C}$ ). These results suggest that endogenous Epe1 is regulated by cAMP signaling. Finally, polysome profiling shows that endogenous $e p e 1^{+}$mRNA is broadly distributed into different fractions in wild-type cells. However, its presence in the polysome fractions is reduced in git $3 \Delta$ cells (Fig $4 \mathrm{D}$ and $4 \mathrm{E}$ ). These results suggest that cAMP signaling also specifically regulates endogenous epe $1^{+}$mRNA translation, irrespective of its expression levels. In contrast, there are minor differences in the distribution of $c l r 4^{+}$mRNA in polysome fractions between wild type and git3 4 cells (S3A Fig), and Clr4 protein levels are not affected by git3 (S3B Fig)

\section{The cAMP signaling pathway regulates Epe1 function in heterochromatin assembly}

Since Epel levels are reduced when the cAMP signaling pathway is inactive, we examined if git3 3 phenocopies epe $1 \Delta$ in heterochromatin regulation. Epe1 was originally identified as a factor required for confining the silent mating-type region heterochromatin within proper boundaries and epe1 $\Delta$ results in the silencing of the SacI::ade6 ${ }^{+}$reporter [25]. In git3 $\Delta$ cells, SacI::ade6 ${ }^{+}$is also silenced, leading to red/pink colonies, although the effect is weaker compared with epe1 $\Delta$ (Fig 5A). RT-qPCR analyses of the ade $^{+}$transcript also show a stronger silencing effect in epe $1 \Delta$ cells than in git $3 \Delta$ cells, and epe $1 \Delta$ git $3 \Delta$ cells behave similarly to epe $1 \Delta$ cells (Fig 5A). In addition, serial dilution and RT-qPCR analyses show that git3 4 cgs $1 \Delta$ reduced heterochromatin spreading at SacI::ade6 ${ }^{+}$compared with git3A (Fig 5A), consistent with Epe1 protein level changes in these cells (Fig 4C).

The fission yeast genome contains a number of small heterochromatin islands that exhibit varying levels of $\mathrm{H} 3 \mathrm{~K} 9 \mathrm{me} 2$ and in epe $1 \Delta$ cells, $\mathrm{H} 3 \mathrm{~K} 9 \mathrm{me} 2$ levels increase at a majority of these islands [5]. ChIP analyses show that $\mathrm{H} 3 \mathrm{~K} 9 \mathrm{me} 2$ levels increase to a similar extent at two major

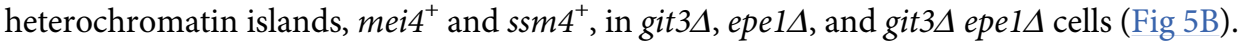

Epe1 also regulates heterochromatin inheritance $[39,40]$. During DNA replication, the passage of the replication fork disrupts parental nucleosomes. Parental $(\mathrm{H} 3-\mathrm{H} 4)_{2}$ tetramers, which are marked by $\mathrm{H} 3 \mathrm{~K} 9 \mathrm{me} 3$, are deposited at the original location and to both daughter strands to direct the formation of nucleosomes. The remaining gaps in DNA are filled by nucleosomes formed with newly synthesized $(\mathrm{H} 3-\mathrm{H} 4)_{2}$. The $\mathrm{H} 3 \mathrm{~K} 9 \mathrm{~m} 3$ on parental histones recruits $\mathrm{Clr} 4$, which contains a chromodomain that recognizes $\mathrm{H} 3 \mathrm{~K} 9 \mathrm{me} 3$. Clr4 then methylates nearby nucleosomes containing newly synthesized histones, therefore restoring the original histone modification profiles on both replicated DNA strands [41]. Since most native heterochromatin regions contain signals for the recruitment of Clr4, ectopic heterochromatin systems have been developed to specifically examine heterochromatin inheritance in the absence of initiation signals [39,40]. For example, when the SET domain of Clr4 is targeted to ten copies of tetO binding sites through a TetR fusion protein (TetR-Clr4-SET), the formation of a large heterochromatin domain silences a neighboring GFP reporter gene $\left(\operatorname{tet} O-g f p^{+}\right)$[40] (S4A Fig). The addition of tetracycline to the medium leads to the quick release of 
A

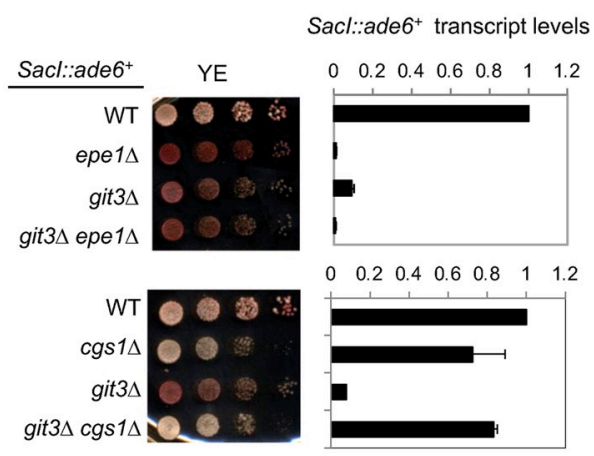

C

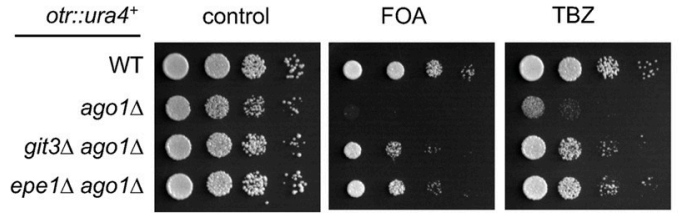

B
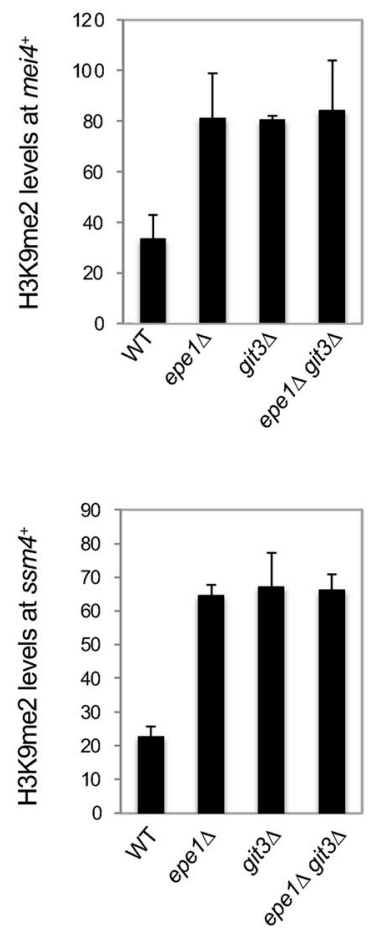

Fig 5. The cAMP signaling pathway regulates heterochromatin formation. (A) Left, Ten-fold serial dilution analyses to measure the expression of the SacI::ade $\sigma^{+}$reporter gene. Right, RT-qPCR analysis of $a d e 6^{+}$transcript, normalized to $a c t 1^{+}$. The endogenous $a d e 6^{+}$in these strains contains an internal deletion (DN/N). Primers were designed to amplify the reporter $a d e 6^{+}$but not the endogenous ade6-DN/N. Error bars represent $S D, n=2$. (B) ChIP analyses of $\mathrm{H} 3 \mathrm{~K} 9 \mathrm{me} 2$ levels at two prominent heterochromatin islands. Error bars represent SD, n =3. (C) Ten-fold serial dilution analyses to measure the expression of otr::ura4 ${ }^{+}$and sensitivity to TBZ.

https://doi.org/10.1371/journal.pgen.1010049.g005

TetR-Clr4-SET. Endogenous Clr4 is recruited to regions with preexisting H3K9me3 and methylates newly incorporated histones due to replication-coupled nucleosome assembly or histone turnover, resulting in the inheritance of this ectopic heterochromatin (S4A Fig). In wild-type cells, this inheritance mechanism is hindered by Epe1-mediated erasure of $\mathrm{H} 3 \mathrm{~K} 9 \mathrm{me}$. As a result, heterochromatin decays quickly, and fluorescence-activated cell sorting (FACS) shows that the expression of GFP gradually increases over a 24-hour period after tetracycline addition (S4B Fig). In epe1 $\Delta$ cells, the majority of cells still silence GFP expression 24 hours after tetracycline addition. However, git $3 \Delta$ results in defective silencing of the tetO-gfp ${ }^{+}$reporter even before the addition of tetracycline (S4B Fig), making it difficult to assess the effects of cAMP signaling in heterochromatin inheritance using this system. The reason that git $3 \Delta$ causes silencing defects at tetO-gfp is unclear. The cAMP signaling pathway may regulate other factors that indirectly affect the silencing at the reporter locus.

To overcome this complication, we examined the effects of the cAMP signaling pathway on the inheritance of pericentric heterochromatin in the absence of RNAi, which is also dependent on the inactivation of Epe1 [41]. For instance, ago1d results in the loss of silencing of the otr::ura4 ${ }^{+}$reporter, and cells are sensitive to microtubule poison thiabendazole (TBZ) due to the requirement of pericentric heterochromatin for chromosome segregation. In epe14 ago $1 \Delta$ cells, both silencing of otr::ura4 ${ }^{+}$and TBZ sensitivity are restored [22,40] (Fig 5C). Consistent with the idea that cAMP signaling regulates Epe1 function, git3 $\Delta$ ago $1 \Delta$ cells also partially rescue otr::ura $4^{+}$silencing defects and TBZ sensitivity associated with agold (Fig 5C) 


\section{Low glucose treatment reduces Epel protein levels and changes heterochromatin landscape}

In fission yeast, the cAMP signaling pathway is active when cells are grown in a rich medium such as YEA (3\% glucose) but inactive when glucose is scarce. Epe1 protein levels decrease after 6 hours of growth in a low glucose medium ( $0.1 \%$ glucose, $3 \%$ glycerol), even though epe $1^{+}$mRNA levels increase (Fig 6A and 6B), suggesting that Epe1 protein levels are controlled by a post-transcriptional mechanism in low glucose conditions as well. In addition, Epe1 protein levels are partially restored in cgs $1 \Delta$ cells subjected to low glucose treatment (S5A and S5B Fig).

We then measured the effects of low glucose medium on heterochromatin functions. qRT-PCR analyses show that 6 hours of low glucose treatment results in about $50 \%$ reduction of SacI::ade6 ${ }^{+}$expression in wild-type cells, suggesting increased heterochromatin spreading.

A

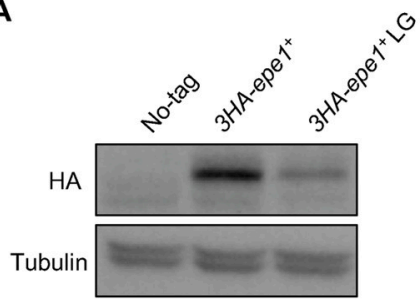

B
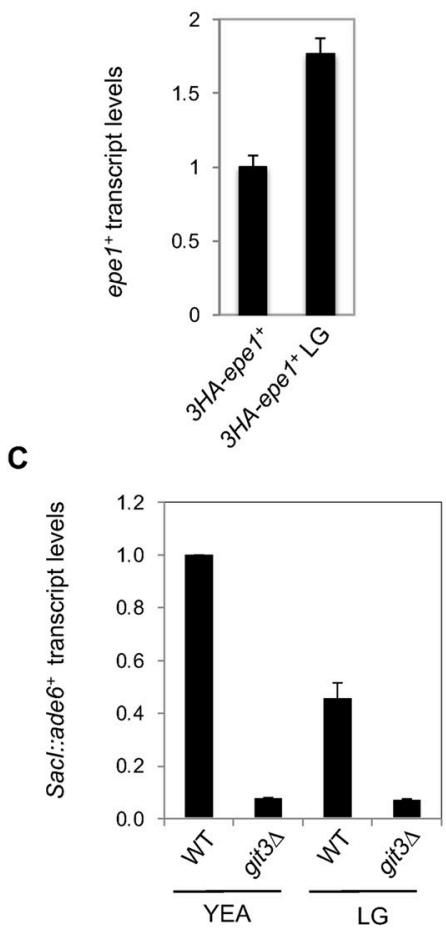

D

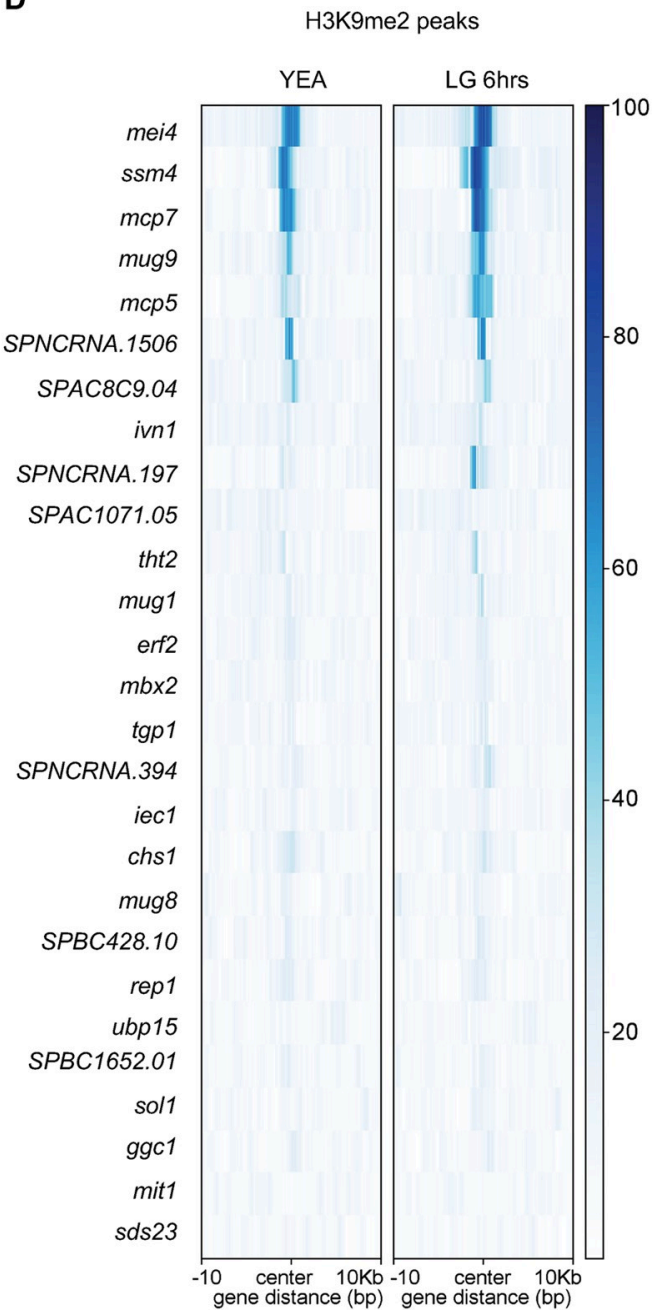

Fig 6. Extracellular glucose concentration affects Epel protein levels and heterochromatin formation. (A) Western blot analysis to measure the levels of 3HA-tagged Epe1 and tubulin. Cells were shifted to a low-glucose medium (LG) for 6 hours before protein extraction. (B) RT-qPCR analysis of epe $1^{+}$transcript levels, normalized to $a_{c t 1}{ }^{+}$. Error bars represent SD, $\mathrm{n}=2$. (C) RT-qPCR analysis of $a d e 6^{+}$transcript, normalized to act $^{+}$. Error bars represent $\mathrm{SD}, \mathrm{n}=2$. (D) Heatmap of $\mathrm{H} 3 \mathrm{~K} 9 \mathrm{me} 2$ levels at individual heterochromatin islands.

https://doi.org/10.1371/journal.pgen.1010049.g006 
In contrast, such treatment has little impact on SacI::ade $6^{+}$expression in git $3 \Delta$ cells, suggesting that the reduction in wild-type cells is due to decreased cAMP signaling (Fig 6C). In addition, FACS analysis shows that heterochromatin at the tetO-gf $\mathrm{p}^{+}$reporter decays slower in cells grown in low glucose medium after tetracycline addition to remove tetR-Clr4-SET (S4C Fig). However, we note that there is decreased silencing of the reporter before tetracycline addition and cells grow slower in low glucose medium, therefore making this result difficult to interpret. Finally, ChIP-seq analyses show that $\mathrm{H} 3 \mathrm{~K} 9 \mathrm{me} 2$ levels at many heterochromatin islands appear to increase in cells grown in low glucose medium for 6 hours than those grown in high glucose medium (YEA) (Fig 6D). These results together suggest that extracellular glucose concentration may regulate heterochromatin functions through Epe1.

\section{Discussion}

In fission yeast, the heterochromatin landscape is regulated by diverse environmental signals such as nutrition and temperature $[5,6,42]$. However, the mechanism behind heterochromatin changes is poorly understood. In this study, we found that the activation of Pkal by the cAMP signaling pathway, in response to high glucose levels, is required to maintain the proper levels of a JmjC domain protein Epe1.

Epel plays an important role in shaping the heterochromatin landscape by serving as the major "eraser" of the H3K9me mark in vivo. Loss of Epel causes heterochromatin expansion, ectopic heterochromatin island formation, and improved heterochromatin inheritance. In contrast, overexpression of Epe1 leads to heterochromatin defects. Therefore, Epe1 protein levels need to be tightly regulated within a narrow range. Indeed, Epe1 is a target of the Cul4-Ddb1 ubiquitin E3 ligase, which mediates its degradation by the proteasome [30]. Our results demonstrate that cAMP signaling affects the translation of the $e p e 1^{+} \mathrm{mRNA}$, independent of protein degradation. Polysome profiling indicates that the translation of $e p e 1^{+} \mathrm{mRNA}$ is less efficient compared with mRNAs of other genes such as the housekeeping gene act $1^{+}$or an inducible gene $n m t 1^{+}$, and more sensitive to disruptions of cAMP signaling. The feature of epe $1^{+}$that is responsible for this translation control seems to be within the epe $1^{+}$coding region, making it challenging to perform further mutational analysis. Interestingly, a recent study shows that in fission yeast, ribosomes stall on tryptophan codons upon oxidative stress [43]. It is possible that the amino acid composition of Epe1 might contribute to the lower translation efficiency.

Previous studies found that heterochromatin islands are affected in low glucose conditions, but with some discrepancies. An earlier study reports an increase of $\mathrm{H} 3 \mathrm{~K} 9 \mathrm{me} 2$ in heterochromatin islands during glucose starvation while a recent study reports a decrease of $\mathrm{H} 3 \mathrm{~K} 9 \mathrm{me} 2$ at heterochromatin islands $[42,44]$. Our ChIP-seq analysis showed that average H3K9me2 levels increase in cells grown in a low glucose medium for 6 hours compared to cells grown in a high glucose medium (Fig 6D). The differences in results could originate from different media conditions, antibodies, or the duration of exposure to low glucose. We also note that our study used glycerol as an alternative carbon source to allow cell growth in the low glucose medium. Our finding that many islands show an increase in $\mathrm{H} 3 \mathrm{~K} 9 \mathrm{me} 2$ is consistent with the reduction of Epe1 levels. However, low glucose likely affects heterochromatin islands in multiple ways besides its effect on Epe1 levels as glucose starvation changes not only cAMP signaling but also the levels of many metabolites, which could affect diverse histone-modifying activities. Therefore, the effects on heterochromatin islands will reflect the sum of diverse contributions.

Recent studies have begun to tackle the mechanistic link between nutrient conditions and changes in heterochromatin. For example, the TOR signaling pathway promotes the stability of Pir1, a component of the RNA elimination machinery involved in facultative 
heterochromatin formation [42]. Our data reveal a new link between glucose sensing, cAMP signaling, and the protein levels of a JmjC protein Epe1. This fits into a growing body of evidence that nutritional conditions not only change cellular metabolite levels but also affect signaling pathways to modify chromatin. Many of these regulatory events are at the posttranscriptional level, which allows cells to quickly respond to stimuli.

\section{Materials and methods}

\section{Fission yeast strains and genetic analyses}

Yeast strain containing $3 H A-e p e 1^{+}$was constructed using the SpEDIT CRISPR method [45].

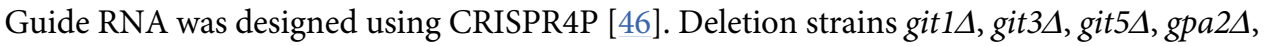
pka1 $1 \Delta$, and $\operatorname{cgs} 2 \Delta$ were derived from the Bioneer deletion library and the absence of the gene coding regions was confirmed by PCR analyses. The strains containing $\operatorname{cgs} 1 \Delta$ or $\operatorname{cyr} 1 \Delta$ were constructed by a PCR-based module method [38]. All other strains were constructed by genetic crosses. A list of yeast strains used is provided in S1 Table. Yeasts were grown in EMM (Edinburgh minimal medium, MPBio, 4110022,) or YEA (Yeast extract with adenine, 0.5\% yeast extract, $3 \%$ glucose, and $100 \mathrm{mg} / \mathrm{l}$ adenine). For glucose deprivation experiments, yeast cells were grown first in YEA medium, washed twice with water, and resuspended in a low glucose medium (YEA with $0.1 \%$ glucose and 3\% glycerol) and grown for 6 hours before ChIP analyses or RNA extraction. For serial dilution plating assays, ten-fold dilutions of a mid-log phase culture were plated on the indicated media and grown for $4-6$ days at $30^{\circ} \mathrm{C}$ for EMMbased plates and 3 days at $30^{\circ} \mathrm{C}$ for other plates.

\section{Screen for suppressors of Epe1 overexpression}

Query strain (nmt41-epe $1^{+}$-natMX6 otr:ura4 $\left.{ }^{+}-h p h M X 6\right)$ was crossed with a library of strains that contains individual gene deletions marked with kanMX6 cassette, using a Singer RoToR HDA pinning robot. The desired haploid progenies, which contain nmt41-epe $1^{+}$-natMX6 otr: $u r a 4^{+}-h p h M X 6$ and a single gene deletion were selected and pinned first onto EMM plates for 1 day to induce nmt41 expression, and subsequently pinned onto EMM plates supplemented with $100 \mu \mathrm{g} / \mathrm{ml} \mathrm{FOA} \mathrm{to} \mathrm{measure} \mathrm{growth.}$

\section{Chromatin immunoprecipitation (ChIP) analyses}

ChIP experiments were performed as described previously [47]. Briefly, log-phase yeast cells were crosslinked with $3 \%$ formaldehyde for 30 minutes at $18^{\circ} \mathrm{C}$ with shaking. Cells were harvested, washed with PBS (phosphate-buffered saline), and resuspended in ChIP lysis buffer (50 mM HEPES-KOH, pH 7.5, 140 mM NaCl, $1 \%$ Triton X-100, 0.1\% Deoxycholate, $1 \mathrm{mM}$ PMSF). Cells were disrupted with glass beads in a bead beater. The lysates were collected, diluted with ChIP lysis buffer, and sonicated with a Bioruptor Pico (Diagenode) for 10 cycles (30s on/30s off) to produce DNA fragments of 100-500 bp in length. The cleared lysates were incubated with the following antibodies overnight at $4^{\circ} \mathrm{C}$ : H3K9me2 (Abcam, 115159), H3K14ac (Upstate, 07-353), and Pol II S2P (Abcam, ab5059). Dynabeads ${ }^{\text {TM }}$ Protein G (Thermo Fisher Scientific, 10004D) was then added to capture the antibodies and associated chromatin fragments. The beads were washed with ChIP lysis buffer twice, ChIP lysis buffer containing $0.5 \mathrm{M} \mathrm{NaCl}$, Wash buffer (10 mM Tris, pH 8.0, $250 \mathrm{mM} \mathrm{LiCl,} \mathrm{0.5 \%} \mathrm{NP-40,} \mathrm{0.5 \%}$ Deoxycholate, $1 \mathrm{mM}$ EDTA), and TE (50 mM Tris pH 8.0, $1 \mathrm{mM}$ EDTA). The bound chromatin fragments were eluted with TES (50 mM Tris pH 8.0, $1 \mathrm{mM} \mathrm{EDTA,} \%$ SDS) at $65^{\circ} \mathrm{C}$ for 10 minutes with shaking. The crosslinking was reversed by incubating at $65^{\circ} \mathrm{C}$ overnight. The protein-DNA mixture was treated with proteinase $\mathrm{K}$, and DNA was purified by phenol: 
chloroform extraction and ethanol precipitation. Quantitative real-time PCR (qPCR) was performed with Luna Universal qPCR Master Mix (NEB, M3003X) in a StepOne Plus Real-Time PCR System (Applied Biosystems). DNA serial dilutions were used as templates to generate a standard curve of amplification for each pair of primers, and the relative concentration of the target sequence was calculated accordingly. A leu1 $1^{+}$or $a c t 1^{+}$fragment was used as a reference to calculate the enrichment of ChIP over WCE for $\mathrm{H} 3 \mathrm{~K} 9 \mathrm{me} 2$. A list of DNA oligos used is provided in S2 Table.

\section{ChIP-seq}

Log-phase yeast cells were crosslinked with $1 \%$ formaldehyde for 20 minutes with shaking at room temperature, followed by 5 minutes quenching with $125 \mathrm{mM}$ glycine. Cells were harvested, washed with PBS (phosphate-buffered saline), and resuspended in ChIP lysis buffer (50 mM HEPES-KOH, pH 7.5, 140 mM NaCl, 1\% Triton X-100, 0.1\% Deoxycholate, $1 \mathrm{mM}$ PMSF). Ice-cold glass beads were added, and the mixtures were vigorously disrupted in a bead-beater with four $30 \mathrm{~s}$ rounds. The lysates were collected, to which NP buffer was added (10 mM Tris, pH 7.4, $1 \mathrm{M}$ sorbitol, $50 \mathrm{mM} \mathrm{NaCl}, 5 \mathrm{mM} \mathrm{MgCl} 2,1 \mathrm{mM} \mathrm{CaCl}$ ). MNase was added to the reaction and the reactions were incubated at $37^{\circ} \mathrm{C}$ for 20 minutes. MNase amount was titrated empirically so that the chromatin was digested to yield mainly mono- and dinucleosomes. The reaction was stopped by adding $10 \mathrm{mM}$ EDTA, and the tubes were placed on ice. $5 \mathrm{X}$ ChIP lysis buffer was added to the reaction, mixed by short vertexing, and the tubes were incubated on ice for 30 minutes. The reactions were then cleared by centrifugation at $16,000 \mathrm{x} g$ for 10 minutes. $4 \%$ of the cleared supernatant was reserved as input and the rest was used for immunoprecipitation. The protocols for immunoprecipitation, reverse-crosslinking, and DNA precipitation were as in the previous ChIP section. The precipitated DNA was treated with RNase A (Thermo Fisher Scientific, EN0531) for 1 hour at $37^{\circ} \mathrm{C}$. DNA concentration was determined with the Qubit dsDNA HS Assay Kit (Thermo Fisher Scientific, Q33230). 5-10 ng of ChIP and input DNA were used for library construction using the NEBNext Ultra II DNA Library Prep Kit for Illumina (NEB, E7645). Libraries were pooled and sequenced on a NextSeq500/550 with the Mid-output kit (150 cycles, single-end) at the JP Sulzberger Genome Center at Columbia University.

Sequencing reads were de-multiplexed and aligned to the $S$. pombe reference genome (ASM294v2), obtained from Pombase [48] with Bowtie2 using default parameters [49]. Peaks were called with MACS2 [50], and only peaks appearing in both replicates were included for downstream analysis. Genome-wide coverage was calculated with deepTools2 [51] and normalized to counts per million (CPM). The two replicates were merged to yield the average track. The coverage plot was visualized with IGV [52]. The heatmap and average profile plot were generated by deepTools2 using the union of peaks present in all strains/conditions. ChIP-seq experiments were performed in duplicates for each genotype.

\section{RNA analyses}

RNA analyses were performed as described [28]. Briefly, RNA was extracted from log-growth phase yeast cultures using MasterPure Yeast RNA Purification Kit (Epicentre). RT-qPCR analyses were performed with Luna Universal One-Step RT-qPCR Kit (NEB, E3005L) in a StepOne Plus Real-Time PCR System (Applied Biosystems). RNA serial dilutions were used as a template to generate the standard curve of amplification for each pair of primers, and the relative concentration of the target sequence was calculated accordingly. An $a c t 1^{+}$fragment served as a reference to normalize the concentration of samples. The concentration of each target in wild type was arbitrarily set to 1 and served as a reference for other samples. 


\section{Protein extraction and western blot analysis}

Protein extraction was performed either using the bead-beating method or a NaOH-TCA method [53]. For the bead-beating method, log-phase yeast cells were harvested and lysed by beads-beating following the same lysis protocol as in ChIP. The resulting $\sim 100 \mu \mathrm{l}$ lysate was diluted with $300 \mu \mathrm{l} \mathrm{ChIP} \mathrm{lysis} \mathrm{buffer} \mathrm{and} \mathrm{mixed} \mathrm{by} \mathrm{vertexing.} \mathrm{An} \mathrm{aliquot} \mathrm{of} \mathrm{the} \mathrm{lysate} \mathrm{was}$ mixed with an equal amount of $2 \mathrm{X}$ SDS loading buffer and boiled for 10 minutes at $80^{\circ} \mathrm{C}$. The boiled lysate was centrifuged at $16,000 \times g$ and $8 \mu l$ supernatant was separated on an SDS-PAGE gel, followed by the transfer of the proteins to a PVDF membrane. The membrane was blotted with antibodies against Tubulin (Gift from Keith Gull) [54] and HA (Santa Cruz, sc7392). The membrane was visualized using a ChemiDoc imaging system (BioRad). For the cycloheximide-chase experiment, cycloheximide was added to the medium to a final concentration of $0.15 \mathrm{mg} / \mathrm{ml} .2 \times 10^{7}$ cells were harvested at the indicated time points and protein was extracted with the $\mathrm{NaOH}-\mathrm{TCA}$ method. Western blot quantification was performed with ImageJ.

\section{Polysome profiling}

Approximately $5 \times 10^{8}$ of yeast cells were lysed using a Fast Prep machine in polysome lysis buffer (20 mM Tris- $\mathrm{HCl} \mathrm{pH} \mathrm{7.5,10} \mathrm{mM} \mathrm{magnesium} \mathrm{chloride,} 50 \mathrm{mM}$ potassium chloride, $10 \mu \mathrm{g} / \mathrm{ml}$ cycloheximide, $1 \mathrm{mM}$ PMSF, 1x Halt protease and phosphatase inhibitor cocktail (ThermoFisher Scientific, 78442)). Lysate was cleared by centrifugation at $4^{\circ} \mathrm{C}$ at $20,000 \mathrm{~g}$ for 10 minutes. Lysate was loaded on a $10 \%$ to $50 \%$ sucrose gradient in polysome lysis buffer. Gradients were centrifuged for 2 hours at 37,000 rpm in a Beckman SW41Ti rotor. Fractions were collected with a BioComp gradient station and a BioComp TRiAX flow cell monitoring continuous absorbance at $260 \mathrm{~nm}$. To each fraction, an equal volume of phenol:chloroform $\mathrm{pH} 4.5$ was added and fractions were flash frozen. For RNA extractions, the fractions were placed in a $65^{\circ} \mathrm{C}$ water bath and vortexed frequently for 30 minutes. The fractions were then extracted twice with phenol-chloroform and once with chloroform, and RNA was extracted with isopropanol precipitation.

\section{In vitro phosphorylation assay}

In vitro phosphorylation was carried out in phosphorylation assay buffer $(25 \mathrm{mM} \mathrm{pH7.5} \mathrm{Tris-}$ $\mathrm{HCl}, 10 \mathrm{mM} \mathrm{MgCl} 2,1 \mathrm{mM}$ Dithiothreitol, $100 \mu \mathrm{M}$ ATP and $5 \mu \mathrm{Ci} \gamma$-32P-ATP) with recombinant Epe1, Pka1 and Rst2 fragment. Reactions were carried out at $30^{\circ} \mathrm{C}$ for 30 minutes with mild shaking. To the reaction, $5 \mu \mathrm{l} 5 \mathrm{X}$ SDS loading buffer was added and incubated at $80^{\circ} \mathrm{C}$ for 10 minutes to stop the reaction. Reactions were separated on an SDS-PAGE gel. The gel was dried in a gel dryer at $80^{\circ} \mathrm{C}$ for 1 hour. The dried gel was exposed to a Phosphor Storage Screen (GE) and the screen was imaged using a Typhoon Imager (GE).

\section{FACS analysis}

Cells containing TetR-Clr4-SET and tetO-ura4-GFP reporter were cultured and kept in logarithm phase, and were harvested at various time points after the addition of tetracycline $(2.5 \mathrm{mg} / \mathrm{ml})$. Cells were collected and fixed by the addition of $70 \%$ ethanol for 20 minutes. The cells were then washed twice with PBS $\left(10 \mathrm{mM} \mathrm{Na}_{2} \mathrm{HPO}_{4}, 1.8 \mathrm{mM} \mathrm{KH}_{2} \mathrm{PO}_{4}, \mathrm{pH} 7.4,137 \mathrm{mM}\right.$ $\mathrm{NaCl}, 2.7 \mathrm{mM} \mathrm{KCl}$ ), and resuspended in a FACS tube (BD Falcon). GFP fluorescence was measured using FACSCelesta (Becton Dickinson), and excitation was achieved by using an argon laser emission of $488 \mathrm{~nm}$. Data collection was performed using Cellquest (Becton Dickinson), and a primary gate based on physical parameters (forward and side light scatter) was set to 
exclude dead cells or debris. Typically, 50,000 cells were analyzed for each sample and time point. Raw data were processed and histograms were drawn using FlowJo (10.6.2, Becton Dickinson).

\section{Supporting information}

S1 Fig. Pka1 phosphorylates Epe1. (A) In vitro kinase assay measuring Pka1's activity towards Epe1 and a positive control Rst2 (1-380). Left, recombinant full length Epe1 purified from insect cells were used. Note that Epe1 is phosphorylated without adding recombinant Pka1, suggesting that insect cell lysates contain a kinase activity that phosphorylates Epe1. Right, recombinant Epe1 fragments purified from E.coli were used. * represents Pka1 autophosphorylation and arrows represent Epe1 fragments. (B) Diagram of Epe1 truncations and their phosphorylation status in (A). (C, D) Western blots to measure Epe1 mutants levels and tubulin. S717 is a phosphorylation site identified by mass-spec analysis of in vitro phosphorylated recombinant Epe1. S606 and T607 are predicted Pka1 phosphorylation sites based on bioinformatics analysis.

S2 Fig. Endogenous HA tagged Epe1 is functional. (A) Diagram of the mating-type region with SacI::ade $\sigma^{+}$reporter gene. The shaded area represents heterochromatin. (B) Ten-fold serial dilution analyses to measure the expression of SacI::ade6 ${ }^{+}$.

S3 Fig. Defective cAMP signalling has no impact on the translation of Clr4. (A) qRT-PCR analysis of $\mathrm{clr}^{+}$transcripts from each fraction of polysome profile. Relative amounts of transcripts were calculated using the delta Ct method. The distribution is shown as a percentage of the total. Error bars represent the SD of two technical replicates. (B) Western blot analysis to measure the levels of endogenous Myc-tagged Clr4 and tubulin.

S4 Fig. The effects of cAMP signaling and extracellular glucose concentration on the inheritance of an ectopic heterochromatin. (A) Diagram of the TetR-Clr4-SET system. The targeting of the SET domain of $\mathrm{Clr} 4$ to tet $\mathrm{O}$ sites results in the formation of heterochromatin and silencing of the adjacent $g f p^{+}$reporter gene. The addition of tetracycline (TET) results in the release of $\mathrm{Clr} 4$ from tet $\mathrm{O}$ sites and heterochromatin is maintained by endogenous $\mathrm{Clr} 4$, which contains a chromodomain that recognizes H3K9me. (B,C) FACS analyses of GFP expression. Samples were taken at indicated time points after the addition of tetracycline. (EPS)

S5 Fig. Extracellular glucose concentration affects Epe1 protein levels. (A) Western blot analyses to measure the levels of 3HA-tagged Epe1 and tubulin. (B) Quantification of Epe1 proteins levels. Error bars represent SD, $\mathrm{n}=3$.

(EPS)

S1 Table. Yeast strains used in this study. (XLSX)

S2 Table. DNA oligos used in this study. (XLSX) 


\section{Acknowledgments}

We thank Karl Ekwall for strains, Rodney Rothstein, Laura Landweber, Marko Jovanovic, and members of the Jia lab for discussions, and Qiulin Zhu for editing the manuscript.

\section{Author Contributions}

Conceptualization: Kehan Bao, Chun-Min Shan, Songtao Jia.

Data curation: Kehan Bao, Chun-Min Shan, Xiao Chen, Gulzhan Raiymbek, Jeremy G. Monroe, Yimeng Fang, Takenori Toda, Kristin S. Koutmou, Kaushik Ragunathan, Luke E. Berchowitz, Songtao Jia.

Funding acquisition: Kristin S. Koutmou, Kaushik Ragunathan, Chao Lu, Luke E. Berchowitz, Songtao Jia.

Investigation: Kehan Bao, Chun-Min Shan, Xiao Chen, Gulzhan Raiymbek, Jeremy G. Monroe, Yimeng Fang, Takenori Toda, Luke E. Berchowitz.

Methodology: Kehan Bao, Chun-Min Shan.

Supervision: Kristin S. Koutmou, Kaushik Ragunathan, Chao Lu, Luke E. Berchowitz, Songtao Jia.

Writing - original draft: Kehan Bao.

Writing - review \& editing: Kehan Bao, Chun-Min Shan, Yimeng Fang, Takenori Toda, Kaushik Ragunathan, Chao Lu, Luke E. Berchowitz, Songtao Jia.

\section{References}

1. Grewal SI, Jia S. Heterochromatin revisited. Nat Rev Genet. 2007; 8(1):35-46. Epub 2006/12/19. nrg2008 [pii] https://doi.org/10.1038/nrg2008 PMID: 17173056.

2. Allshire RC, Madhani HD. Ten principles of heterochromatin formation and function. Nat Rev Mol Cell Biol. 2018; 19(4):229-44. Epub 2017/12/14. https://doi.org/10.1038/nrm.2017.119 PMID: 29235574; PubMed Central PMCID: PMC6822695.

3. Janke R, Dodson AE, Rine J. Metabolism and epigenetics. Annu Rev Cell Dev Biol. 2015; 31:473-96. Epub 2015/09/12. https://doi.org/10.1146/annurev-cellbio-100814-125544 PMID: 26359776; PubMed Central PMCID: PMC5091661.

4. Suganuma T, Workman JL. Chromatin and Metabolism. Annu Rev Biochem. 2018; 87:27-49. Epub 2018/06/22. https://doi.org/10.1146/annurev-biochem-062917-012634 PMID: 29925263.

5. Zofall M, Yamanaka S, Reyes-Turcu FE, Zhang K, Rubin C, Grewal SI. RNA elimination machinery targeting meiotic mRNAs promotes facultative heterochromatin formation. Science. 2012; 335(6064):96100. Epub 2011/12/07. https://doi.org/10.1126/science.1211651 PMID: 22144463.

6. Gallagher PS, Larkin M, Thillainadesan G, Dhakshnamoorthy J, Balachandran V, Xiao H, et al. Iron homeostasis regulates facultative heterochromatin assembly in adaptive genome control. Nat Struct Mol Biol. 2018; 25(5):372-83. Epub 2018/04/25. https://doi.org/10.1038/s41594-018-0056-2 PMID: 29686279; PubMed Central PMCID: PMC5936480.

7. Wang J, Reddy BD, Jia S. Rapid epigenetic adaptation to uncontrolled heterochromatin spreading. eLife. 2015; 4. Epub 2015/03/17. https://doi.org/10.7554/eLife.06179 PMID: 25774602; PubMed Central PMCID: PMC4395908.

8. Torres-Garcia S, Yaseen I, Shukla M, Audergon P, White SA, Pidoux AL, et al. Epigenetic gene silencing by heterochromatin primes fungal resistance. Nature. 2020; 585(7825):453-8. Epub 2020/09/11. https://doi.org/10.1038/s41586-020-2706-x PMID: 32908306; PubMed Central PMCID: PMC7116710.

9. Hall IM, Shankaranarayana GD, Noma K, Ayoub N, Cohen A, Grewal SI. Establishment and maintenance of a heterochromatin domain. Science. 2002; 297(5590):2232-7. Epub 2002/09/07. https://doi. org/10.1126/science.1076466 PMID: 12215653.

10. Volpe TA, Kidner C, Hall IM, Teng G, Grewal SI, Martienssen RA. Regulation of heterochromatic silencing and histone H3 lysine-9 methylation by RNAi. Science. 2002; 297(5588):1833-7. Epub 2002/08/24. https://doi.org/10.1126/science.1074973 PMID: 12193640. 
11. Verdel A, Jia S, Gerber S, Sugiyama T, Gygi S, Grewal SI, et al. RNAi-mediated targeting of heterochromatin by the RITS complex. Science. 2004; 303(5658):672-6. Epub 2004/01/06. https://doi.org/10. 1126/science.1093686 PMID: 14704433; PubMed Central PMCID: PMC3244756.

12. Zhang K, Mosch K, Fischle W, Grewal SI. Roles of the Clr4 methyltransferase complex in nucleation, spreading and maintenance of heterochromatin. Nat Struct Mol Biol. 2008; 15(4):381-8. Epub 2008/03/ 18. https://doi.org/10.1038/nsmb.1406 PMID: 18345014.

13. Bayne EH, White SA, Kagansky A, Bijos DA, Sanchez-Pulido L, Hoe KL, et al. Stc1: a critical link between RNAi and chromatin modification required for heterochromatin integrity. Cell. 2010; 140 (5):666-77. Epub 2010/03/10. https://doi.org/10.1016/j.cell.2010.01.038 PMID: 20211136; PubMed Central PMCID: PMC2875855.

14. Jia S, Noma K, Grewal SI. RNAi-independent heterochromatin nucleation by the stress-activated ATF/ CREB family proteins. Science. 2004; 304(5679):1971-6. Epub 2004/06/26. https://doi.org/10.1126/ science.1099035 PMID: 15218150.

15. Kanoh J, Sadaie M, Urano T, Ishikawa F. Telomere binding protein Taz1 establishes Swi6 heterochromatin independently of RNAi at telomeres. Curr Biol. 2005; 15(20):1808-19. Epub 2005/10/26. https:// doi.org/10.1016/j.cub.2005.09.041 PMID: 16243027.

16. Wang J, Cohen AL, Letian A, Tadeo X, Moresco JJ, Liu J, et al. The proper connection between shelterin components is required for telomeric heterochromatin assembly. Genes Dev. 2016; 30(7):827-39. Epub 2016/03/19. https://doi.org/10.1101/gad.266718.115 PMID: 26988418; PubMed Central PMCID: PMC4826398.

17. Bannister AJ, Zegerman P, Partridge JF, Miska EA, Thomas JO, Allshire RC, et al. Selective recognition of methylated lysine 9 on histone H3 by the HP1 chromo domain. Nature. 2001; 410(6824):120-4. Epub 2001/03/10. https://doi.org/10.1038/35065138 PMID: 11242054.

18. Nakayama J, Rice JC, Strahl BD, Allis CD, Grewal SI. Role of histone H3 lysine 9 methylation in epigenetic control of heterochromatin assembly. Science. 2001; 292(5514):110-3. Epub 2001/04/03. https:// doi.org/10.1126/science.1060118 PMID: 11283354.

19. Sadaie M, lida T, Urano T, Nakayama J. A chromodomain protein, Chp1, is required for the establishment of heterochromatin in fission yeast. Embo J. 2004; 23(19):3825-35. Epub 2004/09/17. https://doi. org/10.1038/sj.emboj.7600401 PMID: 15372076; PubMed Central PMCID: PMC522800.

20. Shan CM, Bao K, Diedrich J, Chen X, Lu C, Yates JR 3rd, et al. The INO80 Complex Regulates Epigenetic Inheritance of Heterochromatin. Cell Rep. 2020; 33(13):108561. Epub 2020/12/31. https://doi.org/ 10.1016/j.celrep.2020.108561 PMID: 33378674; PubMed Central PMCID: PMC7896557.

21. Zofall M, Grewal SI. Swi6/HP1 recruits a JmjC domain protein to facilitate transcription of heterochromatic repeats. Mol Cell. 2006; 22(5):681-92. Epub 2006/06/10. https://doi.org/10.1016/j.molcel.2006. 05.010 PMID: 16762840.

22. Trewick SC, Minc E, Antonelli R, Urano T, Allshire RC. The JmjC domain protein Epe1 prevents unregulated assembly and disassembly of heterochromatin. Embo J. 2007; 26(22):4670-82. Epub 2007/10/ 20. https://doi.org/10.1038/sj.emboj.7601892 PMID: 17948055; PubMed Central PMCID: PMC2048757.

23. Sadaie M, Kawaguchi R, Ohtani $Y$, Arisaka F, Tanaka K, Shirahige K, et al. Balance between distinct HP1 family proteins controls heterochromatin assembly in fission yeast. Mol Cell Biol. 2008; 28 (23):6973-88. Epub 2008/09/24. https://doi.org/10.1128/MCB.00791-08 PMID: 18809570; PubMed Central PMCID: PMC2593388.

24. Tsukada $\mathrm{Y}$, Fang J, Erdjument-Bromage $\mathrm{H}$, Warren $\mathrm{ME}$, Borchers $\mathrm{CH}$, Tempst $\mathrm{P}$, et al. Histone demethylation by a family of JmjC domain-containing proteins. Nature. 2006; 439(7078):811-6. Epub 2005/12/ 20. https://doi.org/10.1038/nature04433 PMID: 16362057.

25. Ayoub N, Noma K, Isaac S, Kahan T, Grewal SI, Cohen A. A novel jmjC domain protein modulates heterochromatization in fission yeast. Mol Cell Biol. 2003; 23(12):4356-70. Epub 2003/05/30. https://doi. org/10.1128/MCB.23.12.4356-4370.2003 PMID: 12773576; PubMed Central PMCID: PMC156127.

26. Wang J, Tadeo X, Hou H, Tu PG, Thompson J, Yates JR, 3rd, et al. Epe1 recruits BET family bromodomain protein Bdf2 to establish heterochromatin boundaries. Genes Dev. 2013; 27(17):1886-902. Epub 2013/09/10. https://doi.org/10.1101/gad.221010.113 PMID: 24013502; PubMed Central PMCID: PMC3778242.

27. Garcia JF, Al-Sady B, Madhani HD. Intrinsic Toxicity of Unchecked Heterochromatin Spread Is Suppressed by Redundant Chromatin Boundary Functions in Schizosacchromyces pombe. G3 (Bethesda). 2015; 5(7):1453-61. Epub 2015/05/10. https://doi.org/10.1534/g3.115.018663 PMID: 25957277; PubMed Central PMCID: PMC4502379.

28. Bao K, Shan CM, Moresco J, Yates J, 3rd, Jia S. Anti-silencing factor Epe1 associates with SAGA to regulate transcription within heterochromatin. Genes Dev. 2019; 33(1-2):116-26. Epub 2018/12/24. https://doi.org/10.1101/gad.318030.118 PMID: 30573453; PubMed Central PMCID: PMC6317313. 
29. Sorida M, Hirauchi T, Ishizaki H, Kaito W, Shimada A, Mori C, et al. Regulation of ectopic heterochromatin-mediated epigenetic diversification by the JmjC family protein Epe1. PLoS Genet. 2019; 15(6): e1008129. Epub 2019/06/18. https://doi.org/10.1371/journal.pgen.1008129 PMID: 31206516; PubMed Central PMCID: PMC6576747.

30. Braun S, Garcia JF, Rowley M, Rougemaille M, Shankar S, Madhani HD. The Cul4-Ddb1(Cdt)(2) ubiquitin ligase inhibits invasion of a boundary-associated antisilencing factor into heterochromatin. Cell. 2011; 144(1):41-54. Epub 2011/01/11. https://doi.org/10.1016/j.cell.2010.11.051 PMID: 21215368; PubMed Central PMCID: PMC3645473.

31. Basi G, Schmid E, Maundrell K. TATA box mutations in the Schizosaccharomyces pombe nmt1 promoter affect transcription efficiency but not the transcription start point or thiamine repressibility. Gene. 1993; 123(1):131-6. Epub 1993/01/15. https://doi.org/10.1016/0378-1119(93)90552-e PMID: 8422997.

32. Allshire RC, Nimmo ER, Ekwall K, Javerzat JP, Cranston G. Mutations derepressing silent centromeric domains in fission yeast disrupt chromosome segregation. Genes Dev. 1995; 9(2):218-33. https://doi. org/10.1101/gad.9.2.218 PMID: 7851795.

33. Hoffman CS. Glucose sensing via the protein kinase A pathway in Schizosaccharomyces pombe. Biochem Soc Trans. 2005; 33(Pt 1):257-60. Epub 2005/01/26. https://doi.org/10.1042/BST0330257 PMID: 15667320; PubMed Central PMCID: PMC4418446.

34. Sassone-Corsi P. The cyclic AMP pathway. Cold Spring Harb Perspect Biol. 2012; 4(12). Epub 2012/ 12/05. https://doi.org/10.1101/cshperspect.a011148 PMID: 23209152; PubMed Central PMCID: PMC3504441.

35. Wang L, Griffiths K Jr., Zhang YH, Ivey FD, Hoffman CS. Schizosaccharomyces pombe adenylate cyclase suppressor mutations suggest a role for CAMP phosphodiesterase regulation in feedback control of glucose/cAMP signaling. Genetics. 2005; 171(4):1523-33. Epub 2005/09/07. https://doi.org/10. 1534/genetics.105.047233 PMID: 16143612; PubMed Central PMCID: PMC1456081.

36. DeVoti J, Seydoux G, Beach D, McLeod M. Interaction between ran1+ protein kinase and cAMP dependent protein kinase as negative regulators of fission yeast meiosis. Embo J. 1991; 10(12):3759-68. Epub 1991/12/01. PMID: 1657594; PubMed Central PMCID: PMC453112.

37. Liu C, Powell KA, Mundt K, Wu L, Carr AM, Caspari T. Cop9/signalosome subunits and Pcu4 regulate ribonucleotide reductase by both checkpoint-dependent and -independent mechanisms. Genes Dev. 2003; 17(9):1130-40. Epub 2003/04/16. https://doi.org/10.1101/gad.1090803 PMID: 12695334; PubMed Central PMCID: PMC196048.

38. Bahler J, Wu JQ, Longtine MS, Shah NG, McKenzie A 3rd, Steever AB, et al. Heterologous modules for efficient and versatile PCR-based gene targeting in Schizosaccharomyces pombe. Yeast. 1998; 14 (10):943-51. Epub 1998/08/26. https://doi.org/10.1002/(SICI)1097-0061(199807)14:10<943::AIDYEA292>3.0.CO;2-Y PMID: 9717240.

39. Audergon PN, Catania S, Kagansky A, Tong P, Shukla M, Pidoux AL, et al. Epigenetics. Restricted epigenetic inheritance of H3K9 methylation. Science. 2015; 348(6230):132-5. Epub 2015/04/04. https:// doi.org/10.1126/science.1260638 PMID: 25838386; PubMed Central PMCID: PMC4397586.

40. Ragunathan K, Jih G, Moazed D. Epigenetics. Epigenetic inheritance uncoupled from sequence-specific recruitment. Science. 2015; 348(6230):1258699. Epub 2015/04/02. https://doi.org/10.1126/ science.1258699 PMID: 25831549; PubMed Central PMCID: PMC4385470.

41. Shan $\mathrm{CM}$, Fang $\mathrm{Y}$, Jia S. Leaving histone unturned for epigenetic inheritance. FEBS J. 2021. Epub 2021/11/03. https://doi.org/10.1111/febs.16260 PMID: 34726351.

42. Wei $\mathrm{Y}$, Lee NN, Pan L, Dhakshnamoorthy J, Sun LL, Zofall M, et al. TOR targets an RNA processing network to regulate facultative heterochromatin, developmental gene expression and cell proliferation Nat Cell Biol. 2021; 23(3):243-56. Epub 2021/02/13. https://doi.org/10.1038/s41556-021-00631-y PMID: 33574613.

43. Rubio A, Ghosh S, Mulleder M, Ralser M, Mata J. Ribosome profiling reveals ribosome stalling on tryptophan codons and ribosome queuing upon oxidative stress in fission yeast. Nucleic Acids Res. 2021; 49(1):383-99. Epub 2020/12/15. https://doi.org/10.1093/nar/gkaa1180 PMID: 33313903; PubMed Central PMCID: PMC7797079.

44. Yamanaka S, Mehta S, Reyes-Turcu FE, Zhuang F, Fuchs RT, Rong Y, et al. RNAi triggered by specialized machinery silences developmental genes and retrotransposons. Nature. 2013; 493(7433):557-60. Epub 2012/11/16. https://doi.org/10.1038/nature11716 PMID: 23151475; PubMed Central PMCID: PMC3554839.

45. Torres-Garcia S, Di Pompeo L, Eivers L, Gaborieau B, White SA, Pidoux AL, et al. SpEDIT: A fast and efficient CRISPR/Cas9 method for fission yeast. Wellcome Open Res. 2020; 5:274. Epub 2020/12/15. https://doi.org/10.12688/wellcomeopenres.16405.1 PMID: 33313420; PubMed Central PMCID: PMC7721064. 
46. Rodriguez-Lopez M, Cotobal C, Fernandez-Sanchez O, Borbaran Bravo N, Oktriani R, Abendroth H, et al. A CRISPR/Cas9-based method and primer design tool for seamless genome editing in fission yeast. Wellcome Open Res. 2016; 1:19. Epub 2017/06/15. https://doi.org/10.12688/wellcomeopenres. 10038.3 PMID: 28612052; PubMed Central PMCID: PMC5445975.

47. Shan CM, Wang J, Xu K, Chen H, Yue JX, Andrews S, et al. A histone H3K9M mutation traps histone methyltransferase Clr4 to prevent heterochromatin spreading. eLife. 2016; 5. Epub 2016/09/21. https:// doi.org/10.7554/eLife.17903 PMID: 27648579; PubMed Central PMCID: PMC5030085.

48. Lock A, Rutherford K, Harris MA, Hayles J, Oliver SG, Bahler J, et al. PomBase 2018: user-driven reimplementation of the fission yeast database provides rapid and intuitive access to diverse, interconnected information. Nucleic Acids Res. 2019; 47(D1):D821-D7. Epub 2018/10/16. https://doi.org/10.1093/ nar/gky961 PMID: 30321395; PubMed Central PMCID: PMC6324063.

49. Langmead B, Trapnell C, Pop M, Salzberg SL. Ultrafast and memory-efficient alignment of short DNA sequences to the human genome. Genome Biol. 2009; 10(3):R25. Epub 2009/03/06. https://doi.org/10. 1186/gb-2009-10-3-r25 PMID: 19261174; PubMed Central PMCID: PMC2690996.

50. Feng J, Liu T, Qin B, Zhang Y, Liu XS. Identifying ChIP-seq enrichment using MACS. Nat Protoc. 2012; 7(9):1728-40. Epub 2012/09/01. https://doi.org/10.1038/nprot.2012.101 PMID: 22936215; PubMed Central PMCID: PMC3868217.

51. Ramirez F, Ryan DP, Gruning B, Bhardwaj V, Kilpert F, Richter AS, et al. deepTools2: a next generation web server for deep-sequencing data analysis. Nucleic Acids Res. 2016; 44(W1):W160-5. Epub 2016/ 04/16. https://doi.org/10.1093/nar/gkw257 PMID: 27079975; PubMed Central PMCID: PMC4987876.

52. Robinson JT, Thorvaldsdottir H, Winckler W, Guttman M, Lander ES, Getz G, et al. Integrative genomics viewer. Nat Biotechnol. 2011; 29(1):24-6. Epub 2011/01/12. https://doi.org/10.1038/nbt.1754 PMID: 21221095; PubMed Central PMCID: PMC3346182.

53. Lawrence CL, Maekawa H, Worthington JL, Reiter W, Wilkinson CR, Jones N. Regulation of Schizosaccharomyces pombe Atf1 protein levels by Sty1-mediated phosphorylation and heterodimerization with Pcr1. J Biol Chem. 2007; 282(8):5160-70. Epub 2006/12/22. https://doi.org/10.1074/jbc.M608526200 PMID: 17182615.

54. Woods A, Sherwin T, Sasse R, MacRae TH, Baines AJ, Gull K. Definition of individual components within the cytoskeleton of Trypanosoma brucei by a library of monoclonal antibodies. J Cell Sci. 1989; 93 (Pt 3):491-500. Epub 1989/07/01. PMID: 2606940. 